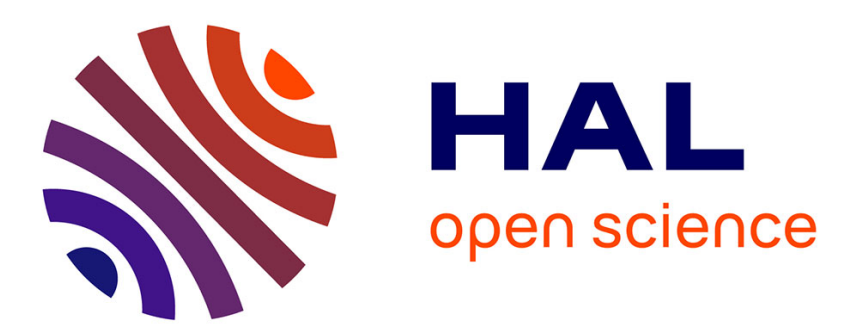

\title{
QPSK super-orthogonal space-time trellis codes with 3 and 4 transmit antennas
}

Pierre Viland, Gheorghe I. Zaharia, Jean-François Hélard

\section{To cite this version:}

Pierre Viland, Gheorghe I. Zaharia, Jean-François Hélard. QPSK super-orthogonal space-time trellis codes with 3 and 4 transmit antennas. Signals, Circuits and Systems, 2011. ISSCS 2011. International Symposium on, Jun 2011, Iasi, Romania. pp.1-4, 10.1109/ISSCS.2011.5978758 hal-00617995

\section{HAL Id: hal-00617995 \\ https://hal.science/hal-00617995}

Submitted on 31 Aug 2011

HAL is a multi-disciplinary open access archive for the deposit and dissemination of scientific research documents, whether they are published or not. The documents may come from teaching and research institutions in France or abroad, or from public or private research centers.
L'archive ouverte pluridisciplinaire HAL, est destinée au dépôt et à la diffusion de documents scientifiques de niveau recherche, publiés ou non, émanant des établissements d'enseignement et de recherche français ou étrangers, des laboratoires publics ou privés. 


\title{
QPSK Super-Orthogonal Space-Time Trellis Codes with 3 and 4 Transmit Antennas
}

\author{
Pierre Viland, Gheorghe Zaharia and Jean-François Hélard \\ Institute for Electronics and Telecommunications of Rennes (IETR) - INSA - UMR CNRS 6164 \\ 20 avenue des Buttes de Coësmes, CS 70839 \\ 35043 Rennes Cedex 7, France \\ Email: pierre.viland@insa-rennes.fr
}

\begin{abstract}
In this paper, we propose, for the first time, QPSK super-orthogonal space-time trellis codes (SO-STTCs) with 3 and 4 transmit antennas and with more than 4 states. In order to decrease drastically the search time of the best SO-STTCs with 3 and 4 transmit antennas, a matrix representation of codes and an adaptation of the coset partitioning are proposed. Thus, we obtain good SO-STTCs with more than 2 transmit antennas. We demonstrate by simulations that the new proposed SO-STTCs outperform the best corresponding STTCs.
\end{abstract}

\section{INTRODUCTION}

Tarokh et al. introduced in [1] the concept of space-time trellis codes (STTCs) to improve the error performance or the date rate for wireless communication systems with multiple transmit and receive antennas. The first design criteria called rank and determinant criteria presented in [1] aim to obtain STTCs achieving full diversity and some coding gain. Chen et al. proposed in [2] the trace criterion to improve the coding gain of STTCs. Based on these criteria, a method called coset partitioning has been proposed in [3] to obtain efficiently the best STTCs.

In [4], Alamouti proposed a simple code achieving full diversity and full rate for two transmit antennas. The generalization of this code leads to a new type of codes [5] called orthogonal space-time block codes (O-STBCs). The main advantages of these codes are to employ a simple decoding scheme and to achieve a full diversity. Nevertheless, O-STBCs do not provide a coding gain. Besides, in the case of complex constellations, there are no O-STBCs with more than two transmit antennas which achieve full diversity and full rate.

Jafarkhani et al. introduced in [6] a new class of codes called super-orthogonal space-time trellis codes (SO-STTCs) for two and four transmit antennas combining O-STBCs and STTCs. In the same time, Ionescu et al. in [7] and Siwamogsatham et al. in [8] proposed similar coding systems to improve the performance of wireless communications. The benefits of SOSTTCs are to provide full diversity compared to STTCs and to improve coding gain compared to O-STBCs. In [9], a simple representation of SO-STTCs with only two transmit antennas based on generator matrices is given to allow a systematic search of all possible codes. For 4 transmit antennas, superquasi-orthogonal space-time trellis codes (SQO-STTCs) have been proposed in [10]. A simplification and a generalization for $2 m$ transmit antennas with $m \in \mathbb{N}^{*}$ of SQO-STTCs are given in [11]. These codes achieve full diversity and full rate. However, SQO-STTCs have the higher coding/decoding complexity compared to SO-STTCs and are proposed for a small number of states.

In this paper, we propose to extend the matrix representation of SO-STTCs [9] for 3 and 4 transmit antennas. This representation allows to design efficiently SO-STTCs, thanks to the coset partitioning [3]. Thus, new SO-STTCs with 3 and 4 transmit antennas are proposed.

The paper is organized as follows. Section II describes the representations of SO-STTCs and the existing design criteria. In section III, the new method is presented. Section IV gives new QPSK SO-STTCs with 3 and 4 transmit antennas. Their performance is compared to that of the best known STTCs.

\section{SYSTEM MODEL}

\section{A. QPSK SO-STTCS}

We consider a QPSK $2^{\nu B_{S}}$-state SO-STTC with $2^{B_{P}}$ parallel branches and $n_{T}$ transmit antennas, where $\nu, B_{S}, B_{P}, n_{T} \in \mathbb{N}^{*}$ and $B_{I}=B_{S}+B_{P}$ is an even number.

The shift register of the encoder is constituted by one input block of $B_{I}$ bits followed by $\nu$ memory blocks of $B_{S}$ bits. Every $T$ symbol-times, $B_{I}$ bits $\left[x_{1}^{t} \cdots x_{B_{I}}^{t}\right]$ are fed into the encoder. The goal of the encoder is to generate $n_{S}=\frac{B_{I}}{2}$ signals every $T$ symbol-times. We define a generator matrix

$$
\mathbf{G}=\left[G_{1}^{1} \cdots G_{B_{I}}^{1}\left|G_{1}^{2} \cdots G_{B_{S}}^{2}\right| \cdots \mid G_{1}^{\nu+1} \cdots G_{B_{S}}^{\nu+1}\right]
$$

where $G_{i}^{l}=\left[g_{1, i}^{l} \cdots g_{n_{S}, i}^{l}\right]^{\mathrm{T}} \in \mathbb{Z}_{4}^{n_{S}}$, where $i=1,2, \cdots, B_{I}$ if $l=1$ and $i=1,2, \cdots, B_{S}$ if $l=2, \cdots, \nu+1$. In this paper, $[\cdot]^{\mathrm{T}}$ is the transpose of the matrix [.]. Besides, we consider the extended state

$$
X^{t}=\left[x_{1}^{t} \cdots x_{B_{I}}^{t}\left|x_{1}^{t-1} \cdots x_{B_{S}}^{t-1}\right| x_{1}^{t-\nu} \cdots x_{B_{S}}^{t-\nu}\right]^{\mathrm{T}}
$$

which is a column vector containing the binary values of the shift register.

Every $T$ symbol-times, the encoder computes

$$
Y=\mathbf{G} X^{t}=\left[y_{1} \cdots y_{n_{S}}\right]^{\mathrm{T}} \in \mathbb{Z}_{4}^{n_{S}} \bmod 4 .
$$

Each encoder output $y_{m}$ with $m=1 \cdots n_{S}$ is mapped onto a QPSK signal given by

$$
s_{m}=e^{j \frac{\pi}{2} y_{m}} .
$$




$$
\begin{gathered}
C_{3}\left(s_{1}, s_{2}, s_{3}, \theta_{1}, \theta_{2}, \theta_{3}\right)=\left[\begin{array}{cccc}
s_{1} e^{j \theta_{1}} & s_{2}^{*} e^{j \theta_{2}} & s_{3}^{*} e^{j \theta_{3}} & 0 \\
-s_{2} e^{j \theta_{1}} & s_{1}^{*} e^{j \theta_{2}} & 0 & s_{3}^{*} \\
-s_{3} e^{j \theta_{1}} & 0 & s_{1}^{*} e^{j \theta_{3}} & s_{2}^{*}
\end{array}\right] \\
C_{4}\left(s_{1}, s_{2}, s_{3}, \theta_{1}, \theta_{2}, \theta_{3}\right)=\left[\begin{array}{cccc}
s_{1} e^{j \theta_{1}} & -s_{2}^{*} e^{j \theta_{2}} & \frac{s_{3}^{*}}{\sqrt{2} e^{j \theta_{3}}} & \frac{s_{3}^{*}}{\sqrt{2}} \\
s_{2} e^{j \theta_{1}} & s_{1}^{*} e^{j \theta_{2}} & \frac{s_{3}^{*}}{\sqrt{2} e^{j \theta_{3}}} & -\frac{s_{3}^{*}}{\sqrt{2}} \\
\frac{s_{3}}{\sqrt{2} e^{j \theta_{1}}} & \frac{s_{3}}{\sqrt{2} e^{j \theta_{2}}} & \frac{-s_{1}-s_{1}^{*}+s_{2}-s_{2}^{*}}{\sqrt{2}} e^{j \theta_{3}} & \frac{s_{2}+s_{2}^{*}+s_{1}-s_{1}^{*}}{\sqrt{2}} \\
\frac{s_{3}}{\sqrt{2}} e^{j \theta_{1}} & -\frac{s_{3}}{\sqrt{2}} e^{j \theta_{2}} & \frac{-s_{2}-s_{2}^{*}+s_{1}-s_{1}^{*}}{\sqrt{2}} e^{j \theta_{3}} & -\frac{s_{1}+s_{1}^{*}+s_{2}-s_{2}^{*}}{\sqrt{2}}
\end{array}\right]
\end{gathered}
$$

During $T$ symbol-times, a codeword of $T$ MIMO symbols is sent over the propagation channel. We consider the codewords given by the expressions (1) proposed in [12] and (2) proposed in [5] in the case of $n_{T}=3$ and $n_{T}=4$ transmit antennas, respectively. For these codes, $n_{S}=3$ and $T=4$.

The goal of multiple rotation angles $\theta_{1}, \theta_{2}$ and $\theta_{3}$ is to increase the number of possible orthogonal codewords. In order to not expand the QPSK constellation, $\theta_{\tau}$ must belong to $\left\{0, \frac{\pi}{2}, \pi, \frac{3 \pi}{2}\right\}$ with $\tau \in\{1,2,3\}$. The $k^{t h}$ line of the expressions (1) and (2) with $k \in\left\{1,2, \cdots, n_{T}\right\}$ corresponds to the signals sent by the $k^{\text {th }}$ transmit antenna. The $t^{t h}$ column of the codewords (1) and (2) with $t \in\{1,2,3,4\}$ corresponds to the MIMO signal transmitted at time $t$ by the $n_{T}$ transmit antennas.

The two codewords of expressions (1) and (2) achieve full rank, but the rates are limited to $3 / 4$. In fact, there are no O-STBCs with full rank and full rate with more than 2 transmit antennas [5]. The rotation angles $\theta_{1}, \theta_{2}$ and $\theta_{3}$ depend on the current state during the transmission of the codeword. For example, the trellis of Fig. 1 describes a QPSK 8-state SOSTTC with 8 parallel paths and 3 transmit antennas. The lines between the points are the transitions between the different states. For each transition, 8 possible codewords can be sent over the propagation channel. The signals $s_{1}, s_{2}$ and $s_{3}$ depend on the inputs bits, the current state and the generator matrix with 3 lines and 9 columns.

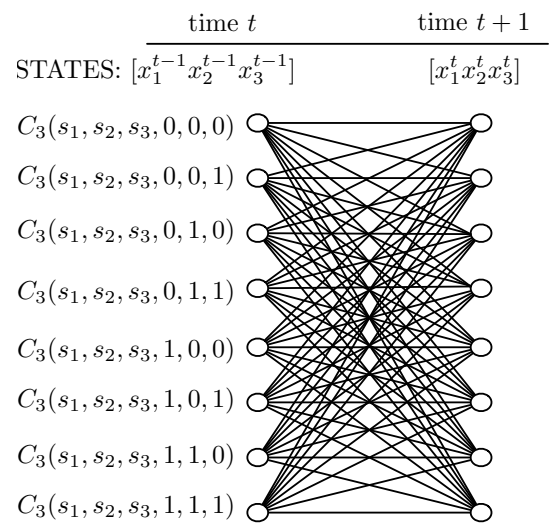

Fig. 1. Trellis representation of QPSK 8-state SO-STTCs with 8 parallel paths and 3 transmit antennas

\section{B. Design criteria}

The main design criteria have been established in [1], [2] in order to decrease the bit and frame error rate. In this paper, only the case of slow Rayleigh fading channels is considered, i.e. the fading coefficients within each frame are constant.

Let us consider a frame of $L$ MIMO signals starting at $t=0$ by a $n_{T} \times L$ matrix $\mathbf{S}=\left[\mathbf{s}^{0} \mathbf{s}^{1} \ldots \mathbf{s}^{L-1}\right]$ where $\mathbf{s}^{q}$ is the $q^{t h}$ MIMO signal. An error occurs if the decoder decides that another frame $\mathbf{E}=\left[\mathbf{e}^{0} \mathbf{e}^{1} \ldots \mathbf{e}^{L-1}\right]$ is transmitted. The main goal of the code design is to reduce the pairwise error probability (PEP) which is the probability that the decoder selects erroneously the frame $\mathbf{E}$ as transmitted frame while the frame $\mathbf{S}$ is actually transmitted. Let define the $n_{T} \times L$ difference matrix $\mathbf{B}=\mathbf{E}-\mathbf{S}$

$$
\mathbf{B}=\left[\begin{array}{ccc}
e_{1}^{0}-s_{1}^{0} & \ldots & e_{1}^{L-1}-s_{1}^{L-1} \\
\vdots & \ddots & \vdots \\
e_{n_{T}}^{0}-s_{n_{T}}^{0} & \cdots & e_{n_{T}}^{L-1}-s_{n_{T}}^{L-1}
\end{array}\right]
$$

The $n_{T} \times n_{T}$ product matrix $\mathbf{A}=\mathbf{B B}^{*}$ is introduced, where $\mathbf{B}^{*}$ denotes the hermitian of $\mathbf{B}$. We define $r=$ $\min (\operatorname{rank}(\mathbf{A}))$, where $\mathbf{A}$ is computed for all pairs of frames $(\mathbf{E}, \mathbf{S})$ with $\mathbf{E} \neq \mathbf{S}$. The design criteria depend on the value of the product $r n_{R}$, where $n_{R}$ is the number of receive antennas.

If $r n_{R} \leq 3$, for a slow Rayleigh fading channel, the rank and determinant criteria have been proposed [1], [13] to reduce the PEP:

- A has to be a full rank matrix for any pair $(\mathbf{E}, \mathbf{S})$.

- The coding gain is related to the inverse of $\eta=$ $\sum_{d} N(d) d^{-n_{R}}$, where $N(d)$ is defined as the average number of error events with determinant $d=\operatorname{det}(\mathbf{A})$. The best codes must have the minimum value of $\eta$.

If $r n_{R} \geq 4$, it is shown in [2] that for a large value of $r n_{R}$ which corresponds to a large number of independent SISO channels, the PEP is minimized if the sum of all the eigenvalues of the matrix $\mathbf{A}$ is maximized. Since $\mathbf{A}$ is a square matrix, the sum of all the eigenvalues is equal to the trace of the matrix $\mathbf{A}$

$$
\operatorname{tr}(\mathbf{A})=\sum_{k=1}^{n_{T}} \lambda_{k}=\sum_{k=1}^{n_{T}}\left(\sum_{q=0}^{L-1}\left|e_{t+q}^{k}-s_{t+q}^{k}\right|^{2}\right) .
$$

For each pair of codewords, $\operatorname{tr}(\mathbf{A})$ is computed. The minimum trace is the minimum of all these values $\operatorname{tr}(\mathbf{A})$. The minimization of the PEP amounts to using a code which has the maximum value of the minimum trace. In [13], it is also stated that to minimize the frame error rate (FER), the number of error events with minimum trace has to be minimized. This criterion is called the trace (or Euclidean distance) criterion. 
TABLE I

QPSK SO-STTCS WITH 3 AND 4 TRANSMIT ANTENNAS

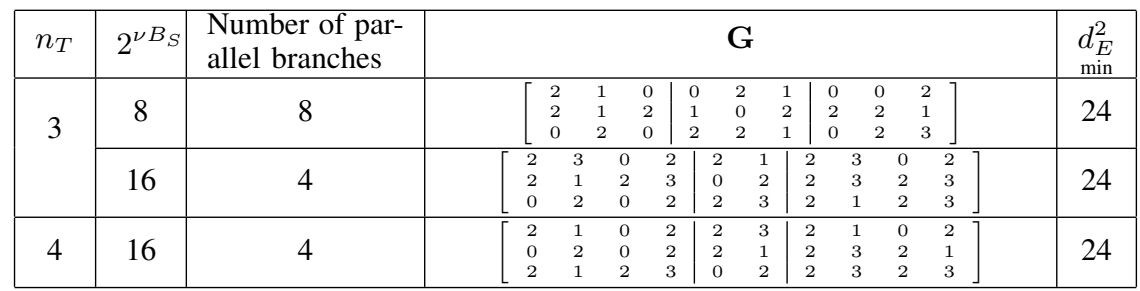

\section{CODE SEARCH}

In the previous publications, in order to maximize the coding gain, the generator matrix was designed via the set partitioning [14] used with the determinant criterion rather than the Euclidean distance criterion. It has been shown in [2] that the Euclidean distance governs the performance of spacetime codes when the number of independent sub-channels is great i.e. $r n_{R} \geq 4$. Thus, we propose to design new SOSTTCs based on the Euclidean distance criterion, rather than the determinant criterion.

As shown for SO-STTCs with 2 transmit antennas in [15] and for STTCs in [3], the generator matrix can be divided into blocks of columns. For example, let us consider the QPSK 8 -state SO-STTCs with 8 parallel branches and 3 transmit antennas where $B_{P}=3$ and $B_{S}=3$. The generator matrix can be represented as

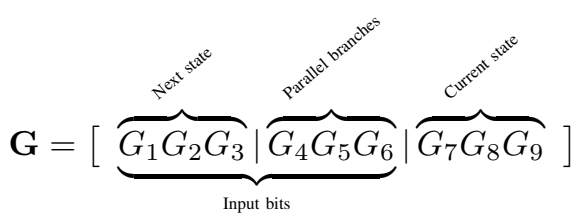

where $G_{l} \in \mathbb{Z}_{4}^{3}$ for $l=1,2, \cdots, 9$. The first block is used to compute the codewords originating from a same state. The second block is employed to compute the codewords of parallel branches of the trellis. Finally, the third block is used to obtain the codewords merging to a same state.

Based on this observation, we adapt the coset partitioning method to design the best SO-STTCs. Thus, the designed codes must fulfill the following rules :

- the used codewords are equally probable.

- the codewords of parallel transitions must be separated by the largest Euclidean distances.

- the MIMO symbols originating from or merging to a same state generate all the possible codewords.

At first, the block which separates the codewords of parallel branches separated by the largest Euclidean distances must be found. The second step is the search of the blocks generating the codewords originating from and merging into the same states as a function of the previous selected block. As does the coset partitioning, the symbols $Y$ originating from the same state must form the group $\mathbb{Z}_{4}^{n_{S}}$. This additive group is the set of all the possible symbols $Y$ given by the expression (5). In fact, since the rotation angles $\theta_{i}$ are fixed for a given state, the number of codewords originating from the same state is equal to $4^{n_{S}}$. Thus, in order to separate by a non-null distance the codewords originating from the same state, the first two blocks must generate all possible codewords. Similarly, the number of codewords merging to the same state is equal to $4^{n_{S}}$. Thus, the last block and the block relative to the parallel branches must generate the group $\mathbb{Z}_{4}^{n_{S}}$. The method to select the columns of generator matrix to obtain a subgroup is presented in [3]. The best codes are combinations of the created blocks.

\section{NEW CODES AND SIMULATION}

Table I presents new QPSK 8-state and 16-state SO-STTCs with 3 and 4 transmit antennas. The number of parallel branches and the minimal Euclidean distance between two codewords (notified $d_{E}^{2}$ ) are given in Table I. Remark : Noexhaustive search has been performed to design the SO-STTCs proposed in Table I.

The performance of each new code is evaluated by simulation in a slow Rayleigh fading channel and compared to the performance of the corresponding STTC in Table II proposed in [3]. For the simulation, the channel fading coefficients are independent samples of a complex Gaussian process with zero mean and variance 0.5 per dimension. These channel coefficients are assumed to be known by the decoder. Each frame consists of 264 bits. For the simulations, we consider 1 and 2 receive antennas. The decoding has two steps. For each transition between two states, the decoder picks the parallel branch with the smallest Euclidean distance. The resulting trellis has only one branch per transition. Then, the Viterbi decoder is applied to find the sent binary data.

TABLE II

STTCS WITH 3 AND 4 TRANSMIT ANTENNAS

\begin{tabular}{|c|c|c|c|c|c|c|c|c|}
\hline$n_{T}$ & $2^{2 \nu}$ & & \multicolumn{5}{|c|}{$\mathbf{G}$} & \multirow{2}{*}{$\frac{d_{\mathrm{min}}^{d_{E}^{2}}}{20}$} \\
\hline \multirow[t]{2}{*}{3} & 8 & $\begin{array}{l}0 \\
2 \\
2\end{array}$ & $\begin{array}{l}2 \\
3 \\
3\end{array}$ & $\begin{array}{l}2 \\
2 \\
2\end{array}$ & $\begin{array}{l}3 \\
3 \\
1\end{array}$ & $\begin{array}{l}0 \\
0 \\
0\end{array}$ & $\begin{array}{l}0 \\
2 \\
2\end{array}$ & \\
\hline & 16 & $\begin{array}{l}0 \\
2 \\
2\end{array}$ & $\begin{array}{l}2 \\
1 \\
1\end{array}$ & $\begin{array}{l}1 \\
2 \\
3\end{array}$ & $\begin{array}{l}2 \\
0 \\
2\end{array}$ & $\begin{array}{l}2 \\
3 \\
1\end{array}$ & $\begin{array}{l}0 \\
2 \\
2\end{array}$ & 24 \\
\hline 4 & 16 & $\begin{array}{l}1 \\
3 \\
2 \\
1\end{array}$ & $\begin{array}{l}2 \\
2 \\
0 \\
2\end{array}$ & $\begin{array}{l}2 \\
3 \\
3 \\
2\end{array}$ & $\begin{array}{l}0 \\
2 \\
2 \\
0\end{array}$ & $\begin{array}{l}3 \\
1 \\
1 \\
2\end{array}$ & $\begin{array}{l}2 \\
2 \\
2 \\
0\end{array}$ & 32 \\
\hline
\end{tabular}

Figs. 2 and 3 present the performance of SO-STTCs and STTCs with 3 transmit antennas presented in Tables I and II with 8 states and 16 states, respectively. Fig. 4 shows the performance of SO-STTCs and STTCs with 4 transmit antennas presented in Tables I and II. In the case of one receive antenna 
and for $\mathrm{FER}=10^{-1}$, according to the simulation results, we can notice that the new proposed SO-STTCs offer a gain of at least $2 \mathrm{~dB}$ over the corresponding best STTCs. In the case of 2 receive antennas and for FER $=10^{-1}$, the SO-STTCs have more than $0.25 \mathrm{~dB}$ of gain compared to the corresponding best STTCs. For 4 transmit antennas, no SQO-STTC with 8 states or more has been proposed in [10], [11].

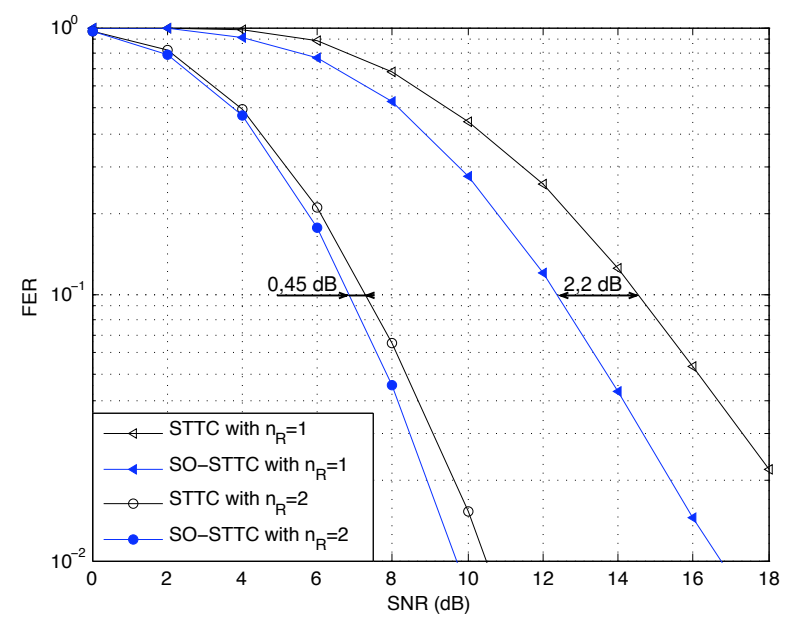

Fig. 2. Performance of the QPSK 8-state STTCs of table II and the QPSK 8-state SO-STTC with 3 transmit antennas

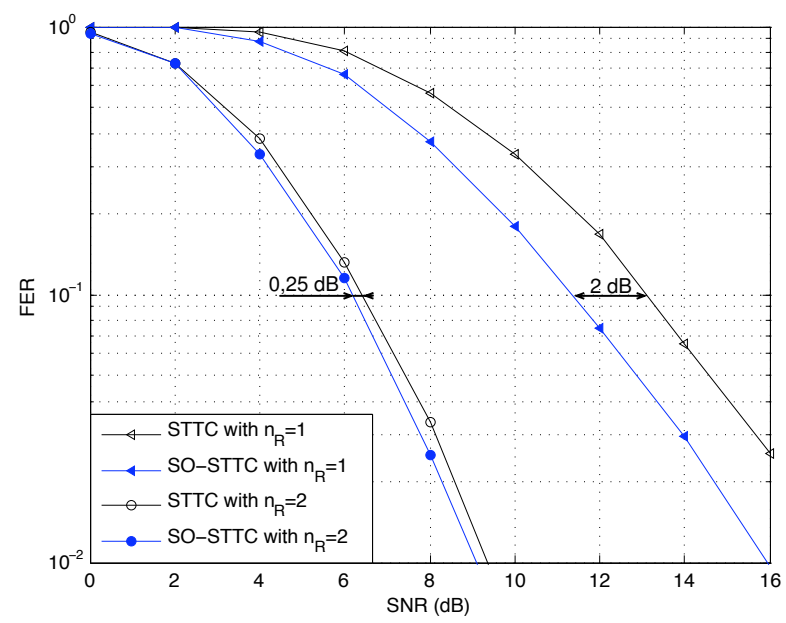

Fig. 3. Performance of the QPSK 16-state STTCs of table II and the QPSK 16-state SO-STTC with 3 transmit antennas

\section{CONCLUSION}

In the paper, the matrix representation of SO-STTCs is extended to the SO-STTCs with 3 and 4 transmit antennas and with more than 4 states. Via this representation and an adaptation of the coset partitioning, good SO-STTCs with 3 and 4 transmit antennas are designed efficiently. Due to the number of transmit antennas and the complex constellation, the new proposed codes do not achieve a full rate. However, they outperform significantly the corresponding STTCs.

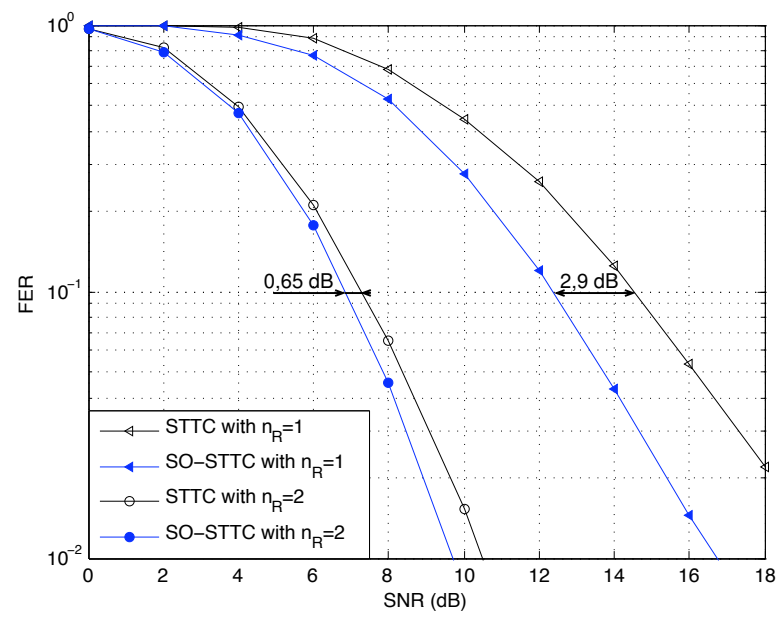

Fig. 4. Performance of the QPSK 16-state STTCs of table II and the SOSTTC with 4 transmit antennas

\section{REFERENCES}

[1] V. Tarokh, N. Seshadri, and A. Calderbank, "Space-time codes for high data rate wireless communication: Performance criterion and code construction," IEEE Trans. on Info. Theory, vol. 44, no. 2, pp. 744-765, Mar. 1998.

[2] Z. Chen, J. Yuan, and B. Vucetic, "Improved space-time trellis coded modulation scheme on slow fading channels," Electron. Lett., vol. 37 no. 7, pp. 440-441, Mar. 2001.

[3] P. Viland, G. Zaharia, and J.-F. Hélard, "Optimal generation of spacetime trellis codes via coset partitioning," IEEE Trans. on Veh. Technol., vol. 60, no. 3, pp. 966-980, Mar. 2011.

[4] S. Alamouti, "A simple transmit diversity technique for wireless communications," IEEE J. on Selected Areas in Comm., vol. 16, no. 8, pp. 1451-1458, Oct. 1998.

[5] V. Tarokh, H. Jafarkhani, and A. Calderbank, "Space-time block codes from orthogonal designs," IEEE Trans. on Info. Theory, vol. 45, no. 5, pp. 1456-1467, Jul. 1999.

[6] H. Jafarkhani and N. Seshadri, "Super-orthogonal space-time trellis codes," IEEE Trans. on Info. Theory, vol. 49, no. 4, pp. 937-950, Apr. 2003.

[7] D. Ionescu, K. Mukkavilli, Y. Zhiyuan, and J. Lilleberg, "Improved 8and 16-state space-time codes for 4PSK with two transmit antennas," IEEE Commun. Lett., vol. 5, no. 7, pp. 301-303, Jul. 2001.

[8] S. Siwamogsatham and M. Fitz, "Improved high-rate space-time codes via concatenation of expanded orthogonal block code and M-TCM," in Proc. IEEE ICC 2002, vol. 1, May 2002, pp. 636-640.

[9] M. Bale, B. Laska, D. Dunwell, F. Chan, and H. Jafarkhani, "Computer design of super-orthogonal space-time trellis codes," IEEE Trans. on Wireless Comm., vol. 6, no. 2, pp. 463-467, Feb. 2007.

[10] H. Jafarkhani and N. Hassanpour, "Super-quasi-orthogonal space-time trellis codes for four transmit antennas," IEEE Trans. on Wireless Comm., vol. 4, no. 1, pp. 215-227, Jan. 2005.

[11] D. Wang, H. Wang, and X.-G. Xia, "Space-time trellis code design based on super quasi-orthogonal block codes with minimum decoding complexity," IEEE Trans. on Comm., vol. 55, no. 8, pp. 1441-1447, Aug. 2007.

[12] B. Hochwald, T. Marzetta, and C. Papadias, "A transmitter diversity scheme for wideband cdma systems based on space-time spreading," IEEE J. on Selected Areas in Comm., vol. 19, no. 1, pp. 48-60, Jan. 2001.

[13] C. Liao and V. Prabhu, "Improved code design criteria for space-time codes over quasi-static flat fading channels," in Proc. IEEE SPAWC 2005, June 2005, pp. 7-11.

[14] G. Ungerboeck, "Channel coding with multilevel/phase signals," IEEE Trans. on Info. Theory, vol. 28, pp. 55-67, Jan. 1982.

[15] E. Rivera Hartling, F. Chan, and H. Jafarkhani, "Design rules for extended super-orthogonal space-time trellis codes," in Proc. IEEE CCECE 2008, May 2008, pp. 1621-1626. 